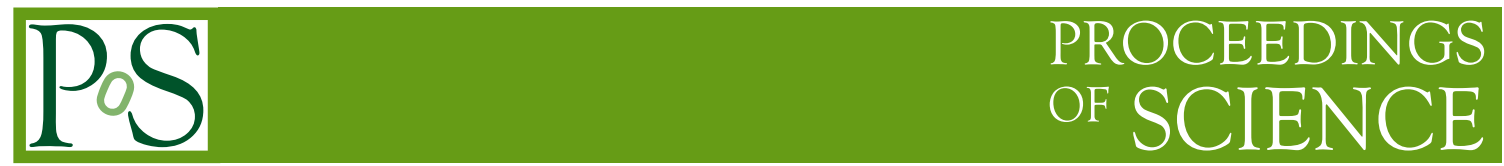

\title{
Search for lepton flavour violation with the ATLAS detector
}

\section{Wing Sheung Chan, on behalf of the ATLAS collaboration*}

Nikhef National Institute for Subatomic Physics, Netherlands

E-mail: ws.chan@ern.ch

\begin{abstract}
Charged lepton flavour violation is an unambiguous signal of beyond-the-Standard-Model phenomena. The search for lepton flavour violation with the ATLAS detector is reported in searches that focus on decays of the $Z$ boson, Higgs boson, $\tau$ lepton and some hypothetical heavy neutral particles or objects, including the extended gauge bosons, R-parity-violating supersymmetry sneutrinos and quantum black holes, using $p p$ collisions data with centre-of-mass energies of 8 $\mathrm{TeV}$ and $13 \mathrm{TeV}$.
\end{abstract}

The 20th International Workshop on Neutrinos (NuFact2018)

12-18 August 2018

Blacksburg, Virginia

${ }^{*}$ Speaker. 
It is well known that flavour-changing processes exist in the quark and neutrino sectors in the Standard Model (SM). However, the mixing of charged leptons have not yet been observed so far. In the SM with neutrino oscillations considered, lepton-flavour-violating (LFV) processes are possible beyond tree level, but have vanishingly small branching ratios. As an example, the LFV $Z \rightarrow \mu \tau$ decay has a branching ratio $\sim 10^{-54}$ [1] in the SM, making it impossible to detect with current experiments. Nonetheless, beyond-the-Standard-Model (BSM) theories such as theories with heavy neutrinos, extended gauge fields and supersymmetry predict such processes to have much higher branching ratios. This makes LFV processes an unambiguous signal of BSM phenomena.

Various searches for lepton flavour violation have been performed with the ATLAS detector [2]. These include searches for LFV decays of SM particles, such as $Z \rightarrow e \mu, Z \rightarrow \ell \tau(\ell=e / \mu)$, $H \rightarrow \ell \tau$ and $\tau \rightarrow 3 \mu$. Decays of some hypothetical heavy neutral particles or objects are also considered, which include the heavy neutral gauge bosons $\left(Z^{\prime}\right)$, the R-parity violating (RPV) sneutrinos $(\tilde{v})$ and quantum blackholes (QBH). $20.3 \mathrm{fb}^{-1}$ of $p p$ collision data at a centre-of-mass energy of $8 \mathrm{TeV}$ collected in the year 2012 (Run- 1 dataset) and $36.1 \mathrm{fb}^{-1}$ of $p p$ collision data at a centreof-mass energy of $13 \mathrm{TeV}$ collected in the years 2015 and 2016 (mid-Run-2 dataset) are used in these searches. In this article, the methods used by and the results of these searches are summarised.

Search for $\boldsymbol{Z} \rightarrow \boldsymbol{e} \boldsymbol{\mu}$ A search for the LFV $Z \rightarrow e \mu$ decay has been performed using the Run-1 dataset [3]. The major SM backgrounds for the analysis are $Z \rightarrow \tau \tau, W W$ and $t \bar{t}$ events. A cutbased analysis approach is used for the search. Events with high-energy jets or with large transverse missing energy $\left(E_{\mathrm{T}}^{\text {miss }}\right)$ are rejected to suppress the $t \bar{t}$ and $W W$ backgrounds. After the event selection, the expected $e \mu$ invariant mass spectrum is fitted to data. Data agree with SM expectation and the upper limit on the branching ratio is set at $95 \%$ confidence level $(\mathrm{CL}): \mathscr{B}(Z \rightarrow e \mu)<7.5 \times 10^{-7}$.

Search for $Z \rightarrow \ell \tau$ Searches for the LFV $Z \rightarrow \ell \tau$ decays using the mid-Run-2 dataset have also been conducted [4]. The analysis focuses on channels with a hadronically-decaying $\tau$ lepton in the final state. Major backgrounds for the analysis include $Z \rightarrow \tau \tau, W+$ jets and $Z \rightarrow \ell \ell$. The $W+$ jets events are important contribution to the total background due to misidentification of quarkor gluon-initiated jets as visible products of hadronic $\tau$ decays ( $\tau_{\text {had-vis }}$ ), hereafter referred to as "fakes". The fakes background is estimated by a data-driven method where the fake rate of the leading- $p_{\mathrm{T}} \tau_{\text {had-vis }}$ candidate is measured using data in dedicated fakes-enriched control regions. $Z \rightarrow \ell \ell$ events contribute to the total background mainly due to misidentification of electrons or muons as $\tau_{\text {had-vis }}$ candidates. In order to suppress the $Z \rightarrow \tau \tau$ and $W+$ jets backgrounds, only events with a low transverse mass of the $\tau_{\text {had-vis }}$ candidate and the $E_{\mathrm{T}}^{\text {miss }}\left(m_{\mathrm{T}}\left(\tau_{\text {had-vis }}, E_{\mathrm{T}}^{\text {miss }}\right)\right)$ are selected. Furthermore, events with a visible invariant mass close to the $Z$ boson rest mass are rejected to reduce the $Z \rightarrow \ell \ell$ background.

The analysis mainly relies on neural network $(\mathrm{NN})$ classifiers to differentiate signal events from the major backgrounds. The classifiers are trained using Monte Carlo (MC) simulated samples and use kinematic variables of the reconstructed $\ell, \tau_{\text {had-vis }}$ and $E_{\mathrm{T}}^{\mathrm{miss}}$ as input. Binned maximumlikelihood fits of the NN classifier output distributions to data in the signal region are performed. Data agree with SM expectation and upper limits on the LFV branching ratios are set at 95\% CL: $\mathscr{B}(Z \rightarrow e \tau)<5.8 \times 10^{-5}$ and $\mathscr{B}(Z \rightarrow \mu \tau)<2.4 \times 10^{-5}$. The results for the $\mu \tau$ channel has been 
combined with previously published ATLAS results using the Run-1 dataset [5] to obtain a more stringent limit: $\mathscr{B}(Z \rightarrow \mu \tau)<1.3 \times 10^{-5}$.

Search for $\boldsymbol{H} \rightarrow \ell \boldsymbol{\tau}$ Searches for $H \rightarrow \ell \tau$ decays have been performed using the Run-1 dataset [6, 7]. Both channels with hadronically-decaying and leptonically-decaying $\tau$ leptons are considered.

For the channels with hadronically-decaying $\tau$ leptons, cuts on $m_{\mathrm{T}}\left(\tau_{\text {had-vis }}, E_{\mathrm{T}}^{\text {miss }}\right)$ and $m_{\mathrm{T}}\left(\ell, E_{\mathrm{T}}^{\text {miss }}\right)$ are used to suppress the major backgrounds, $Z \rightarrow \tau \tau$ and $W+$ jets. In the signal region, the expected distribution of the $\ell \tau$ invariant mass is fitted to data.

For the channels with leptonically-decaying $\tau$ leptons, a different technique is used. Only events with a $e \mu$ final state are considered. Events are classified into two categories, one with the transverse momentum of the electron larger than that of the muon, and vice versa. SM backgrounds in the two categories are expected to be symmetric. However, the signal events are asymmetric in the two categories because the electron or muon that originates from the $\tau$ lepton decay is expected to carry a much lower momentum. Therefore the LFV signal is expected to appear only in one of the two categories. The mass spectra in both categories are simultaneously fitted to data.

Results from the different channels are statistically combined. Data agree with SM expectation and the upper limits are set at 95\% CL: $\mathscr{B}(H \rightarrow e \tau)<1.04 \times 10^{-2}$ and $\mathscr{B}(Z \rightarrow \mu \tau)<1.43 \times 10^{-2}$.

Search for $\tau \rightarrow 3 \mu$ A search for $\tau \rightarrow 3 \mu$ decays has been performed using the Run-1 dataset [8]. The analysis uses $\tau$ leptons from $W \rightarrow \tau \nu$ decays, that have high transverse momentum in general and would therefore decay into muons with momenta high enough to fire the triggers in the case of a signal event. Moreover, the signature recoil $E_{\mathrm{T}}^{\text {miss }}$ from the neutrino helps to reduce backgrounds. Due to the unique signature of having three muons with a common vertex that are close in angle and have an invariant mass close to the $\tau$ lepton rest mass, the signal region of the analysis is almost background-free. Event classification is mainly based on output of boosted decision trees (BDT) trained with MC signal samples and background samples obtained from data in the signal region sidebands. Input to the BDT includes kinematic variables of the three muons and the $E_{\mathrm{T}}^{\text {miss }}$, as well as the vertex fitting probability and significance. Data agree with SM expecation and upper limit on the branching ratio is set at $90 \%$ CL by counting the number of observed events in the signal region: $\mathscr{B}(\tau \rightarrow 3 \mu)<3.76 \times 10^{-7}$.

Search for $Z^{\prime} / \tilde{v} / \mathrm{QBH} \rightarrow \boldsymbol{e} \boldsymbol{\mu} / \boldsymbol{e} \tau / \boldsymbol{\mu} \tau$ Searches for the hypothetical $Z^{\prime}, \tilde{v}$ or QBH into a pair of different-flavour charged leptons have been performed using the mid-Run-2 dataset [9]. For the $e \tau$ and $\mu \tau$ channels, only hadronically-decaying $\tau$ leptons are considered. Major SM backgrounds include $t \bar{t}$ events and, in the channels with $\tau$ leptons, fakes from $W+$ jets and multijet events. The major challenge of the analysis is the estimation of backgrounds in the high-invariant-mass regions. The $t \bar{t}$ background is estimated by fitting smooth functions in the low-invariant-mass regions and extrapolating them. The $W+$ jets and multijet backgrounds are both estimated by data-driven methods where the event or object fake rates are measured using data in dedicated control regions and applied to the signal region. Expected invariant mass spectra are fitted to data and Bayesian lower limits at 95\% credibility level are set on the invariant (threshold) masses of the hypothetical particles (QBHs). Data agree with SM expectation. The observed limits are summarised in Table 1. 
Table 1: Lower limits on the invariant or threshold masses of the considered hypothetical particles or objects at $95 \%$ credibility level.

\begin{tabular}{llll}
\hline \hline \multirow{2}{*}{ Particle or object (model) } & \multicolumn{4}{c}{ Lower limit [TeV] } \\
& $e \mu$ & $e \tau$ & $\mu \tau$ \\
\hline$Z^{\prime}$ (additional $U(1)^{\prime}$ symmetry) & 4.5 & 3.7 & 3.5 \\
$\tilde{v}$ (RPV supersymmetry) & 3.4 & 2.9 & 2.6 \\
QBH (ADD model, $n=6)$ & 5.6 & 4.9 & 4.5 \\
QBH (RS model, $n=1)$ & 3.4 & 2.9 & 2.6 \\
\hline \hline
\end{tabular}

Conclusion Searches of LFV processes are performed with the ATLAS detector using $p p$ collision data at centre-of-mass energies of $8 \mathrm{TeV}$ and $13 \mathrm{TeV}$. LFV decays of the SM particles $Z, H$ and $\tau$, as well as that of the hypothetical particles or objects $Z^{\prime}, \tilde{v}$ and $\mathrm{QBH}$, are considered. No evidence of LFV processes have been found so far. Exclusion limits on the decay branching ratios or masses of the concerned particles or objects are set, which are useful in constraining relevant BSM theories. There are still ongoing efforts in improving these analyses which, together with the increased amount of data collected in the years 2017, 2018 and in the future, are going to further improve the sensitivities of these searches.

\section{References}

[1] J. I. Illana, M. Jack and T. Riemann, Predictions for $Z \rightarrow \mu \tau$ and related reactions, in 5th Workshop of the 2nd ECFA - DESY Study on Physics and Detectors for a Linear Electron - Positron Collider Obernai, France, October 16-19, 1999, pp. 490-524, 1999, hep-ph/ 0001273.

[2] ATLAS Collaboration, The ATLAS experiment at the CERN Large Hadron Collider, JINST 3 (2008) S08003.

[3] ATLAS Collaboration, Search for the lepton flavor violating decay $Z \rightarrow e \mu$ in pp collisions at $\sqrt{s}=8$ TeV with the ATLAS detector, Phys. Rev. D 90 (2014) 072010 [1408. 5774].

[4] ATLAS Collaboration, Search for the lepton flavor violating decay $Z \rightarrow e \mu$ in pp collisions at $\sqrt{s}=8$ TeV with the ATLAS detector, Phys. Rev. D 98 (2018) 092010 [1804. 09568].

[5] ATLAS Collaboration, Search for lepton-flavour-violating decays of the Higgs and Z bosons with the ATLAS detector, Eur. Phys. J. C 77 (2017) 70 [1604.07730].

[6] ATLAS Collaboration, Search for lepton-flavour-violating $H \rightarrow \mu \tau$ decays of the Higgs boson with the ATLAS detector, JHEP 11 (2015) 211 [1508.03372].

[7] ATLAS Collaboration, Search for lepton-flavour-violating decays of the Higgs and Z bosons with the ATLAS detector, Eur. Phys. J. C 77 (2017) 70 [1604.07730].

[8] ATLAS Collaboration, Probing lepton flavour violation via neutrinoless $\tau \rightarrow 3 \mu$ decays with the ATLAS detector, Eur. Phys. J. C 76 (2016) 232 [1601.03567].

[9] ATLAS Collaboration, Search for lepton-flavor violation in different-flavor, high-mass final states in pp collisions at $\sqrt{s}=13$ TeV with the ATLAS detector, Phys. Rev. D 98 (2018) 092008 [1807. 06573]. 